\title{
An application of the SMAA-Choquet method to evaluate the performance of sailboats in offshore regattas
}

\author{
Silvia Angilella ${ }^{1}$, Sally Giuseppe Arcidiacono ${ }^{1}$, Salvatore Corrente ${ }^{1}$, Salvatore Greco $^{1,2}$, and \\ Benedetto Matarazzo ${ }^{1}$
}

${ }^{1}$ Department of Economics and Business, University of Catania, Corso Italia, 55, 95129 Catania, Italy, e-mail: \{angisil,s.arcidiacono, salvatore.corrente, salgreco,matarazz\}@unict.it;

${ }^{2}$ University of Portsmouth, Portsmouth Business School, Centre of Operations Research and Logistics (CORL), Portsmouth PO1 3DE, United Kingdom

\begin{abstract}
In this paper we apply a recently introduced Multiple Criteria Decision Aiding method, namely the SMAA-Choquet method, to compare the performances of different sailboats in regattas. In sailing races where sailboats with different design can participate, the performances of the boats can be evaluated by using different scoring options. In each scoring option, a corrected time is computed taking into account the physical characteristics and the performances of the sailboats. While, in real competitions, the final ranking of the sailboats is obtained by using only one of the considered scoring options, in this paper we propose to aggregate the time values computed by these scoring options in a unique one. Since the time values computed by the scoring options are given on different scales and a certain degree of interaction between them could be observed, we apply the SMAA-Choquet method that is able to deal with both aspects simultaneously.
\end{abstract}

Keywords: Multiple Criteria Decision Aiding, Decision support systems, Sports, interacting criteria, Choquet integral.

\section{Introduction}

In Multiple Criteria Decision Aiding (MCDA; for an introduction to MCDA see Figueira et al. 2016 and Ishizaka and Nemery 2013), a set of $m$ alternatives $A=\left\{a_{1}, a_{2}, \ldots, a_{m}\right\}$ are preferentially compared taking into account a family of $n$ points of view, tacnically called criteria $G=\left\{g_{1}, g_{2}, \ldots, g_{n}\right\}$. In the following, we shall use the term criterion $g_{i}$ or criterion $i$, indifferently. Each criterion can be considered as a function $g_{i}: A \rightarrow \mathbb{R}$ where the performance of $a_{k} \in A$ on criterion $g_{i} \in G$ is denoted by $g_{i}\left(a_{k}\right)$. Moreover, the 
criterion $g_{i}$ can have an increasing or a decreasing direction of preference. In the first case, the higher $g_{i}\left(a_{k}\right)$ the better $a_{k}$ while, in the second case, the higher $g_{i}\left(a_{k}\right)$ the worse $a_{k}$.

The only objective information stemming from the evaluations of the alternatives on the considered criteria is the dominance relation ${ }^{1}$ but, in general, it is really poor. For this reason, these evaluations need to be aggregated to deal with the problem at hand. In literature, three different methodologies are used to perform this aggregation: the Multiple Attribute Value Theory (MAVT; Keeney and Raiffa, 1993), the outranking methods (Brans and Vincke, 1985; Roy, 1996) and the decision rule approach (Greco et al., 2001). In MAVT, a real value is assigned to each alternative being representative of its goodness with respect to the considered problem; outranking methods are based on a pairwise preference relation, denoted by $S$, such that $a_{h} S a_{k}$ if $a_{h}$ is at least as good as $a_{k}$; the decision rule approach provides robust recommendations on the problem at hand by means of "if,..., then..." decision rules expressed in a simple and natural language for the Decision Maker (DM). As shown in Keeney and Raiffa (1993), the value function can be expressed in an additive way if the set of evaluation criteria is mutually preferentially independent. But, in real world applications the evaluation criteria can present a certain degree of positive or negative interaction. For example, supposing to evaluate a sport car with respect to criteria maximum speed, acceleration and price; on one hand, maximum speed and acceleration can be considered as negatively interacting criteria, while, on the other hand, maximum speed and price can be considered as positively interacting criteria. Indeed, a car having high maximum speed has, in general, also a high acceleration. Therefore, even if the two criteria are really important for a DM liking sport cars, the importance assigned to this pair of criteria should be lower than the sum of the importance assigned to the two criteria considered alone. Conversely, a car having a high maximum speed but a low price is well appreciated by the DM since it is not usual and, consequently, the importance assigned to this pair of criteria should be greater than the sum of the importance assigned to the two criteria taken singularly.

In order to aggregate the evaluations of the alternatives on criteria that present a certain degree of interaction, non-additive integrals are used in MCDA and, in particular, the Choquet integral (see Choquet (1953) for the first paper on the Choquet integral and Grabisch (1996) for the application of the Choquet integral in MCDA) and the Sugeno integral (Sugeno, 1974). Two drawbacks of the Choquet integral preference model are represented by the necessity of having the evaluations of the alternatives on the considered criteria expressed on a common scale, and the great number of parameters needed for its application. These two drawbacks have been considered separately in several papers (see Modave and Grabisch (1998) and Angilella et al. (2004) for the construction of a common scale and Angilella et al. (2004) and Marichal and Roubens (2000) for the inference of preference parameters in the Choquet integral). Among the papers that treat these two problems simultaneously, let us remember Corrente et al. (2016) and Angilella et al. (2015). In

\footnotetext{
${ }^{1}$ Supposing that all criteria have an increasing direction of preference, $a_{h}$ dominates $a_{k}$ if $g_{i}\left(a_{h}\right) \geq g_{i}\left(a_{k}\right)$ for all $i=1, \ldots, n$, and there exists at least one $i \in\{1, \ldots, n\}$ such that $g_{i}\left(a_{h}\right)>g_{i}\left(a_{k}\right)$
} 
Corrente et al. (2016), the authors use the Analytical Hierarchy Process (AHP, see e.g. Saaty, 1990) to build the common scale and the Non Additive Robust Ordinal Regression (Angilella et al., 2010) to consider the whole set of capacities compatible with the preferential information supplied by the DM. Angilella et al. (2015) present a new method, namely SMAA-Choquet, proposing an heuristic to build a common scale and using the Stochastic Multiobjective Acceptability Analysis (SMAA; see Lahdelma et al. (1998) for the first paper on SMAA and Tervonen and Figueira (2008) for a survey on SMAA methods) to take into account all common scales and all instances of the Choquet integral preference model compatible with the preferences of the DM.

Sport provides a natural setting for interesting decision making problems and the first applications of the Operation Research tools on this issue have occurred in the 50's (Gerchak, 1994; Mottley, 1954; Wright, 2009). Even if the greatest part of contributions in literature are related to the scheduling of different sport championships (for a survey of scheduling in sports see Kendall et al. 2010), many proposals regarding the application of MCDA to sport can be found as well. In Soares de Mello et al. (2013), the authors apply the MACBETH method (Bana e Costa and Vansnick, 1994) to rank the performances of Brazilian athletes in 2008 Olympic Games; in Dey et al. (2011) the AHP method is applied to compare cricket bowlers in the Indian premier league, while Dadelo et al. (2014) use the TOPSIS method (Hwang and Yoon, 1981) to evaluate the performances of basketball players of the Lithuanian league; Olson (2001) measures the accuracy of PROMETHEE (Brans and Vincke, 1985), SMART (Edwards, 1977) and a centroid method (Barron and Barrett, 1996) in describing the abilities of baseball teams in the American major league. Finally, Jablonsky (2012) proposes several models to aggregate the results obtained by the athletes in the 10 decathlon events analyzing, at the same time, the aggregated results obtained by the PROMETHEE II method.

In this paper we propose to apply the SMAA-Choquet method Angilella et al. (2015) to evaluate the performances of different sailboats in sailing regattas. This method is taken into account, instead of other possible methods, mainly because it permits to deal with interactions between criteria, expressed in different scales, also considering robustness concerns. More precisely:

- through the use of the Choquet integral, it permits to handle positive and negative interactions between criteria,

- through the construction of a common scale for the evaluations on the considered criteria, it permits to aggregate criteria of heterogeneous nature,

- through the consideration of a plurality of preference parameters for the Choquet integral (common scales and sets of non-additive weights called capacities), it permits to handle robustness concerns in multicriteria aggregation.

In general, sailboats of different design (shape, size, etc.) compete in worldwide regattas. Obviously, the design of the sailboats strongly influences their performances, so that comparing them taking into account 
the time necessary to complete the course is not fair. Indeed, for example, long sailboats have an advantage over short ones in case of strong wind, while short sailboats are favored in case of low wind. The scoring options used to rank the participants to the regattas, assign a "corrected time" to each sailboat taking into account the time spent to complete the race and the specific design of each sailboat. Nevertheless, the way in which the scoring options compute this corrected time are based on different assumptions and, consequently, the complete ranking of the participants varies with respect to the considered scoring option.

In this paper, we propose to aggregate the corrected times computed by the different scoring options to assign a single comprehensive corrected time to each sailboat taking into account the good characteristics of each scoring option. To this aim, we can restate this problem as an MCDA problem, by considering the sailboats as alternatives, the different scoring options as the evaluation criteria on which the different alternatives have to be evaluated and, consequently, the corrected time assigned to each sailboat by the considered scoring option as the evaluation of the alternative on the criterion at hand. Since the corrected time values computed by the scoring options are not expressed on the same scale and some type of interaction can be observed among the scoring options, we shall evaluate the comprehensive performance of the different sailboats by using the SMAA-Choquet method.

The paper is organized as follows. In Section 2 we present the five scoring options belonging to the Offshore Racing Congress (ORC). ${ }^{2}$ In Section 3, we recall the Choquet integral preference model, the SMAA methodology and the SMAA-Choquet method, while, in Section 4, we apply the SMAA-Choquet method to the "53th Syracuse - Malta" regatta. ${ }^{3}$ Section 5 collects some conclusions and further directions of research.

\section{Sailboats handicapping rules: the ORC}

Longer sailboats are inherently faster than shorter ones, and so, in the interest of fairness, since the 1820 s in the sailboat races, the larger sailboat started to be imprecisely handicapped. Unfortunately, the results showed to have many problems with the allowance system. Modern rating systems are based on a precise analysis of very specific physical parameters (such as: length, sail area, sail material, etc.) and this analysis is done in a unique way, differently from the calculation of the corrected time on which the final ranking of the sailboats depends. The most prevalent handicap rating systems today are the ORC, the Offshore Racing Rule (ORR), ${ }^{4}$ the IRC ${ }^{5}$ and the Performance Handicap Racing Fleet (PHRF). ${ }^{6}$

The ORC is an international body for the sport of competitive sailing and it is responsible for fixing and maintaining the rating and classification standards used to define offshore, sailboat racing handicap categories. In the ORC handicap system, the final ranking can be established by using a certain number

\footnotetext{
${ }^{2}$ Official website of the ORC: http://www.orc.org

${ }^{3}$ Official website of the Syracuse-Malta regatta: http://www.rmyc.org/races/archive/?id=115\&page=4

${ }^{4}$ http://offshoreracingrule.org/

${ }^{5}$ https://www.rorcrating.com/

${ }^{6}$ http://www.ussailing.org/racing/offshore-big
} 
of scoring options but, usually, none of them is universally accepted or always adopted. This disagreement exists because each of these scoring options takes into account different conditions of the race and, therefore, it is meaningful according to some aspects of the race such as course distance, wind direction, wind strength, etc. In order to make comparable the performances of the different sailboats, each of these scoring options transforms the real time spent by a sailboat to finish the race in another corrected time, taking into account also the different physical characteristics of the sailboat.

The ORC gives a certificate to each sailboat providing the parameters necessary to compute the corrected time in each of the considered scoring options. For any of the simple scoring options, parameters are given for the offshore (coastal/long distance) and for the inshore (windward/leeward) races. In the ORC, there are four main scoring options through which it is possible to build the final ranking and these are the Time on Distance (ToD), the Time on Time (ToT), the Performance Line (PL), and the Triple Number (ToT:low, Medium, Hight). Moreover, in our application, we shall take into account also another scoring option, the General Purpose Handicap method, being a scoring method universally recognized in this sector. The selection of the scoring option usually depends on the size of the sailboats, type and level of the fleet, type of the race and local racing conditions. Moreover, the choice of the scoring option is at the discretion of National Authorities or local event organizers. Figure 1 shows an extract of a sailboat's ORC certificate where we can find the parameters necessary to compute the corrected time, while Table 1 shows the formulas used to obtain the corrected time in each of the five scoring options. The term Distance appearing in the formulas in Table 1 is the distance between the start and the end points of the course expressed in miles.

Figure 1: Example of an ORC certificate ${ }^{7}$

\begin{tabular}{|c|c|c|c|c|c|}
\hline \multirow{2}{*}{\multicolumn{2}{|c|}{$\begin{array}{l}\text { BOAT } \\
\text { Name LOW NOISE } \\
\text { Sail Nr ITA-15911 }\end{array}$}} & \multirow{2}{*}{\begin{tabular}{|l|} 
GPH \\
621.1
\end{tabular}} & \multicolumn{3}{|l|}{ HULL } \\
\hline & & & \multicolumn{3}{|c|}{$\begin{array}{r}\text { Length Overall } 11.2 \\
\text { Maximum Beam } 3.47\end{array}$} \\
\hline \multicolumn{3}{|l|}{ GENERAL } & \multicolumn{3}{|c|}{$\begin{array}{r}\text { Displacement } \quad \mathbf{5 , 8 4} \\
\text { Draft } 1.99\end{array}$} \\
\hline \multicolumn{2}{|c|}{ Class M 37} & & \multicolumn{2}{|c|}{$\begin{array}{r}\text { MS Reg. Division } \\
\text { Dynamic Allow ance }\end{array}$} & Crui \\
\hline \multicolumn{2}{|c|}{$\begin{array}{l}\text { Designer COSSUTTI MAURIZIO } \\
\text { Builder } 2 \text { घM ME M ARINE }\end{array}$} & & $\begin{array}{r}\text { Dyna } \\
\text { Fw d A }\end{array}$ & Fw d Accommodatio & $\begin{array}{l}0.07 \\
\text { Yes }\end{array}$ \\
\hline \multirow{2}{*}{\multicolumn{2}{|c|}{$\begin{array}{r}\text { Series } 08.2006 \\
\text { Age } 04.2008\end{array}$}} & & \multirow{3}{*}{\multicolumn{2}{|c|}{$\begin{array}{r}\text { Hull Construction } \\
\text { Carbon Rudde } \\
\text { Crew Arm Extension }\end{array}$}} & Cor \\
\hline & & & & & No \\
\hline \multirow{2}{*}{\multicolumn{3}{|c|}{$\begin{array}{l}\text { Age Allow ance } 0.292 \% \\
\text { Offset File M 37M K11.OFF - 28.6.2013. 7:51:32 }\end{array}$}} & & & \\
\hline & & & \multicolumn{3}{|c|}{\begin{tabular}{|ccc} 
IMS L & 10.176 VCGD & 0.1 \\
RL & 8.480 VCGM & 0.1
\end{tabular}} \\
\hline \multicolumn{6}{|c|}{ SCORING OPTIONS } \\
\hline & \multicolumn{3}{|c|}{$\begin{array}{c}\text { OFFSHORE } \\
\text { COASTAL / LONG DISTANCE }\end{array}$} & \multicolumn{2}{|c|}{$\begin{array}{r}\text { INSF } \\
\text { WINDWARD }\end{array}$} \\
\hline Time On Distance & \multicolumn{3}{|c|}{604.4} & \multicolumn{2}{|r|}{67} \\
\hline Time On Time & \multicolumn{3}{|c|}{0.9928} & \multicolumn{2}{|r|}{0.9} \\
\hline Performance Line & $\begin{array}{c}\text { PLT } \\
0.830\end{array}$ & \multicolumn{2}{|r|}{$\begin{array}{l}\text { PLD } \\
91.6\end{array}$} & \multicolumn{2}{|l|}{$\begin{array}{l}\text { PLT } \\
\mathbf{0 . 7 9 1}\end{array}$} \\
\hline Triple Number & $\begin{array}{l}\text { Low } \\
0.9354\end{array}$ & $\begin{array}{l}\text { Medium } \\
1.2329\end{array}$ & $\begin{array}{l}\text { High } \\
1.3949\end{array}$ & $\begin{array}{l}\text { Low } \\
0.7134\end{array}$ & $\begin{array}{l}\text { Mec } \\
0.9\end{array}$ \\
\hline
\end{tabular}


Table 1: Formulas for Corrected Times through ORC simple scoring option

\begin{tabular}{|l|l|}
\hline Scoring option & Formula \\
\hline Time on Distance & Elapsed Time $-(T o D \times$ Distance $)$ \\
\hline Time on Time & Elapsed Time $\times$ ToT \\
\hline Performance Line & Elapsed Time $\times$ PLT - PLD $\times$ Distance \\
\hline Triple Number & Elapsed Time $\times$ ToT $($ Low, Medium, High $)$ \\
\hline General Purpouse Handicap & Elapsed Time $-(G P H \times$ Distance $)$ \\
\hline
\end{tabular}

For example, considering the certificate shown in Figure 1 and the five scoring options described in Table 1 , supposing that the time (expressed in seconds) necessary to the sailboat for concluding the offshore course is $t$ and the covered distance is $d$, then the corrected time in the five scoring options is computed as follows:

- Time on Distance: $t-604.4 d$,

- Time on Time: $t \times 0.9928$,

- Performance Line: $t \times 0.83-91.6 \times d$,

- Triple Number: $t \times c$ with $c=0.9354,1.2329,1.3949$ The parameter $c$ for which the elapsed time has to be multiplied is chosen in the end of the course from the authorities or organizers depending on the speed of the wind. If the speed of the wind is Low, then $t$ has to be multiplied by $c=0.9354$, if it is Medium, by $c=1.2329$ and, if it is High, by $c=1.3949$,

- General Purpose Handicap: $t-621.1 d$.

Now, let us show how the application of the different scoring options affects the final ranking of the alternatives. Let us consider three sailboats (XP-ACT, PROFILO, MAYDA) whose parameters necessary to compute the corrected time are shown in Table 2.

Table 2: Parameters necessary for the computation of the corrected time for the five considered scoring options; $t$ is expressed in seconds

\begin{tabular}{|c|ccccccc|}
\hline Sailboat / Paramaters / Real Time & ToD & ToT & PLT & PLD & TN: Low; Medium; High & GPH & $t$ \\
\hline XP-ACT & 542.50 & 1.11 & 0.972 & 116.70 & $1.0593 ; 1.3851 ; 1.5609$ & 624.70 & 76,873 \\
PROFILO & 605.80 & 0.99 & 0.881 & 124.30 & $0.9503 ; 1.2368 ; 1.3952$ & 615.20 & 84,321 \\
MAYDA & 592.80 & 1.01 & 0.882 & 114.40 & $0.9629 ; 1.2638 ; 1.4246$ & 598.50 & 83,188 \\
\hline
\end{tabular}

\footnotetext{
${ }^{7}$ http://www.orc.org/rules/ORC\%20Rating\%20Systems\%202015.pdf
} 
Table 3: Corrected time of the three sailboats with respect to the five scoring options; the considered distance is $d=82.4$ miles, while $t$ is expressed in seconds

\begin{tabular}{|c|ccccc|}
\hline Sailboat / Scoring Option & ToD & ToT & PL & TN & GPH \\
\hline XP-ACT & 32,171 & 85,014 & 65,104 & 102,633 & 31,504 \\
PROFILO & 34,403 & 83,520 & 64,044 & 100,688 & 33,629 \\
MAYDA & 34,341 & 84,195 & 63,945 & 101,248 & 33,872 \\
\hline
\end{tabular}

Using each of the five scoring options, we computed the corrected time for the three sailboats shown in Table 3.

Analyzing the values of the corrected time, it is possible to compute the relative ranking of the three sailboats for each scoring option as shown in Table 4.

Table 4: Ranking of the three sailboats with respect to the five scoring options

\begin{tabular}{|c|ccccc|}
\hline Sailboat / Scoring Option & ToD & ToT & PL & TN & GPH \\
\hline XP-ACT & $1^{\text {st }}$ & $3^{r d}$ & $3^{r d}$ & $3^{\text {rd }}$ & $1^{\text {st }}$ \\
PROFILO & $3^{r d}$ & $1^{\text {st }}$ & $2^{\text {nd }}$ & $1^{\text {st }}$ & $2^{\text {nd }}$ \\
MAYDA & $2^{\text {nd }}$ & $2^{\text {nd }}$ & $1^{\text {st }}$ & $2^{\text {nd }}$ & $3^{\text {rd }}$ \\
\hline
\end{tabular}

As one can see, each of the three sailboats can fill the first position, depending on the considered scoring option. Moreover, we can also underline that:

- XP-ACT fills the first position in two of the considered scoring options, while in the remaining three, it is in the third position,

- PROFILO fills the first position in two of the considered scoring options, the second position in other two scoring options and the third position in the remaining one,

- MAYDA fills the first and the third position for one score option only, while it is in the second position for the other three score options.

\section{A brief reminder of the Choquet integral, the SMAA methodology and the SMAA-Choquet method}

In this paper, we shall apply the SMAA-Choquet method that has been recently introduced in literature (Angilella et al., 2015). It combines the potentials of the Choquet integral preference model and of the SMAA methodology that we shall briefly recall in the next sections. 


\subsection{The Choquet integral}

The Choquet integral preference model (Choquet, 1953) is based on the concept of fuzzy measure, or capacity, being a set function $\mu: 2^{G} \rightarrow[0,1]$, such that $\mu(T)$ represents the comprehensive importance of criteria in $T$ for each $T \subseteq G$, and the following boundary and monotonicity conditions are satisfied:

1a) $\mu(\emptyset)=0, \mu(G)=1$ (boundary conditions),

2a) $\forall R \subseteq T \subseteq G, \mu(R) \leq \mu(T)$ (monotonicty condition).

Given $R, T \subseteq G$ such that $R \cap T=\emptyset$, three cases can occur:

case 1) $\mu(R \cup T)=\mu(R)+\mu(T)$,

case 2) $\mu(R \cup T)>\mu(R)+\mu(T)$,

case 3) $\mu(R \cup T)<\mu(R)+\mu(T)$.

In case 1 ), the sets of criteria $R$ and $T$ are not interacting since the importance assigned to the set of criteria $R \cup T(\mu(R \cup T))$ is equal to the sum of the importance assigned to the two subsets of criteria considered separately $(\mu(R)+\mu(T))$; in case 2$)$, the sets of criteria $R$ and $T$ are positively interacting since the importance assigned to the set of criteria $R \cup T(\mu(R \cup T))$ is greater than the sum of the importance assigned to the two subsets of criteria considered separately $(\mu(R)+\mu(T))$, while in case 3$)$ the sets of criteria $R$ and $T$ are negatively interacting since the importance assigned to the set of criteria $R \cup T(\mu(R \cup T))$ is lower than the sum of the importance assigned to the two subsets of criteria considered separately $(\mu(R)+\mu(T))$.

Given the capacity $\mu$, the Choquet integral assigns to each alternative $a_{k} \in A$ the following value:

$$
C_{\mu}\left(a_{k}\right)=\sum_{i=1}^{n}\left[g_{(i)}\left(a_{k}\right)-g_{(i-1)}\left(a_{k}\right)\right] \cdot \mu\left(A_{i}\right),
$$

where $_{(\cdot)}$ stands for a permutation of the indices of criteria such that $0=g_{(0)}\left(a_{k}\right) \leq g_{(1)}\left(a_{k}\right) \leq \ldots \leq g_{(n)}\left(a_{k}\right)$, $A_{i}=\{(i), \ldots,(n)\}$.

A meaningful and useful reformulation of the capacity $\mu$ and of the Choquet integral can be obtained by means of the Möbius representation of the capacity $\mu$, which is a function $m: 2^{G} \rightarrow \mathbb{R}$ (Rota 1964) defined as follows:

$$
\mu(R)=\sum_{T \subseteq R} m(T)
$$

The Möbius representation $m(R)$ can be obtained from $\mu(R)$ as follows:

$$
m(R)=\sum_{T \subseteq R}(-1)^{(|R \backslash T|)} \mu(T) .
$$


In terms of the Möbius representation of the capacity $\mu$, properties $\mathbf{1 a}$ ) and $\mathbf{2 a}$ ) can be restated as follows (Chateauneuf and Jaffray, 1989)

1b) $m(\emptyset)=0, \sum_{T \subseteq G} m(T)=1$

2b) $\forall g_{i} \in G$ and $\forall R \subseteq G \backslash\left\{g_{i}\right\}, m\left(\left\{g_{i}\right\}\right)+\sum_{T \subseteq R} m(T) \geq 0$

while the Choquet integral may be written as (Gilboa and Schmeidler, 1994):

$$
C_{\mu}\left(a_{k}\right)=\sum_{T \subseteq G} m(T) \cdot \min _{g_{i} \in T} g_{i}\left(a_{k}\right) .
$$

Since the application of the Choquet integral preference model involves the knowledge of $2^{|G|}-2$ parameters (because $\mu(\emptyset)=0$ and $\mu(G)=1$ ), it is advisable using $k$-additive measures (Grabisch, 1997). A fuzzy measure is $k$-additive if $m(T)=0$ for each $T \subseteq G$ such that $|T|>k$. In other words, considering $k$-additive measures we do not take into account the interactions between more than $k$ criteria. In real world applications, 2-additive measures are considered. In this case, the application of the Choquet integral can be applied knowing $n+\left(\begin{array}{l}n \\ 2\end{array}\right)$ values that are, one for each criterion $g_{i}$ and one for each pair of criteria $\left\{g_{i}, g_{j}\right\}$. Considering 2-additive measures, properties $\mathbf{1 b}$ ) and $\mathbf{2 b}$ ) become

1c) $m(\emptyset)=0, \sum_{g_{i} \in G} m\left(\left\{g_{i}\right\}\right)+\sum_{\left\{g_{i}, g_{j}\right\} \subseteq G} m\left(\left\{g_{i}, g_{j}\right\}\right)=1$,

2c) $\left\{\begin{array}{l}m\left(\left\{g_{i}\right\}\right) \geq 0, \forall g_{i} \in G, \\ m\left(\left\{g_{i}\right\}\right)+\sum_{g_{j} \in T} m\left(\left\{g_{i}, g_{j}\right\}\right) \geq 0, \forall g_{i} \in G \text { and } \forall T \subseteq G \backslash\left\{g_{i}\right\}, T \neq \emptyset .\end{array}\right.$

Finally we arrive at the Möbius representation of the Choquet integral with a 2-additive measure for the alternative $a_{k}$

$$
C_{\mu}\left(a_{k}\right)=C_{\mu}\left(g\left(a_{k}\right)\right)=\sum_{g_{i} \in G} m\left(\left\{g_{i}\right\}\right) g_{i}\left(a_{k}\right)+\sum_{\left\{g_{i}, g_{j}\right\} \subseteq G} m\left(\left\{g_{i}, g_{j}\right\}\right) \min \left\{g_{i}\left(a_{k}\right), g_{j}\left(a_{k}\right)\right\}
$$

where $g\left(a_{k}\right)=\left(g_{1}\left(a_{k}\right), g_{2}\left(a_{k}\right), \ldots, g_{n}\left(a_{k}\right)\right)$.

In this context, the importance of criterion $g_{i} \in G$ does not depend only itself, but also on its contribution to all coalitions of criteria. For this reason, the Shapley value (Shapley 1953) is used to represent the importance of a criterion. Considering the 2-additive Möbius representation of a capacity $\mu$, the Shapley value can be expressed as follows:

$$
\varphi\left(\left\{g_{i}\right\}\right)=m\left(\left\{g_{i}\right\}\right)+\sum_{g_{j} \in G \backslash\left\{g_{i}\right\}} \frac{m\left(\left\{g_{i}, g_{j}\right\}\right)}{2} .
$$


Analogously, the Murofushi-Soneda index (Murofushi and Soneda 1993) expresses the importance of the pair of criteria $\left\{g_{i}, g_{j}\right\}$ and in case of the 2-additive Möbius representation of a capacity $\mu$, it can be computed as follows:

$$
\varphi\left(\left\{g_{i}, g_{j}\right\}\right)=m\left(\left\{g_{i}, g_{j}\right\}\right)
$$

\section{$3.2 \quad$ SMAA}

Stochastic Multicriteria Acceptability Analysis (SMAA) (Lahdelma et al., 1998) is a family of methods for aiding multicriteria group decision making in problems with uncertain, imprecise, or partially missing information. Depending on the model considered for representing the preferences of the DM, different SMAA methods have been proposed over the years (Tervonen and Figueira, 2008). Here, we shall recall the SMAA-2 method (Lahdelma and Salminen, 2001) since, in this method, the assumed preference model is a value function and the Choquet integral preference model can be considered as a particular value function.

To take into account imprecision or uncertainty, SMAA-2 considers two probability distributions $f_{\mathcal{M}}(\mu)$ and $f_{\chi}(\xi)$ on $\mathcal{M}$ and $\chi$, respectively, where

$$
\mathcal{M}=\left\{\mu: 2^{G} \rightarrow[0,1]: \mu(\emptyset)=0, \mu(G)=1, \text { and } \mu(R) \leq \mu(T) \text { for all } R \subseteq T \subseteq G\right\}
$$

is the set of admissible capacities and $\chi \subseteq \mathbb{R}^{m \times n}$ is the evaluation space. For each $\mu \in \mathcal{M}$ and for each $\xi \in \chi$, a complete ranking of the alternatives at hand can be computed. SMAA-2 introduces a ranking function relative to the alternative $a_{k}$,

$$
\operatorname{rank}(k, \xi, w)=1+\sum_{h \neq k} \rho\left(C_{\mu}\left(\xi_{h}\right)>C_{\mu}\left(\xi_{k}\right)\right),
$$

where $\rho($ false $)=0, \rho($ true $)=1$ and $\xi_{k}=\left(\xi_{k 1}, \ldots, \xi_{k n}\right) \in \mathbb{R}^{1 \times n}$ is the vector of the performances of alternative $a_{k} \in A$. Consequently, since the position filled by an alternative depends on the capacity $\mu$, for each alternative $a_{k} \in A$, for each performance matrix $\xi \in \chi$ and for each $\operatorname{rank} r=1, \ldots, m$, SMAA-2 computes the set of capacities for which alternative $a_{k}$ assumes rank $r$ :

$$
\mathcal{M}_{k}^{r}(\xi)=\{\mu \in \mathcal{M}: \operatorname{rank}(k, \xi, w)=r\}
$$

SMAA methodologies are therefore based on the computation of the following indices:

- The rank acceptability index, which gives the probability that the alternative $a_{k}$ obtains the rank $r$ :

$$
b_{k}^{r}=\int_{\xi \in \chi} f_{\chi}(\xi) \int_{\mu \in \mathcal{M}_{k}^{r}(\xi)} f_{\mathcal{M}}(\mu) d \mu d \xi
$$


$b_{k}^{r}$ is computed as the frequency with which alternative $a_{k}$ fills rank $k$ in a certain random sampling of preference parameters. It assumes values in the interval $[0,1]$. Obviously, the best alternatives are those presenting an high rank acceptability index for the first positions and a low rank acceptability indices in correspondence of the last positions in the ranking.

- The pairwise winning index $p_{h k}$ (Leskinen et al., 2006) measuring the frequency that an alternative $a_{h}$ is preferred to alternative $a_{k}$ in the space of preference parameters:

$$
p_{h k}=\int_{\mu \in \mathcal{M}} f_{\mathcal{M}}(\mu) \int_{\xi \in \chi: C_{\mu}\left(\xi_{h}\right)>C_{\mu}\left(\xi_{k}\right)} f_{\chi}(\xi) d \xi d \mu .
$$

From a computational point of view, Monte Carlo method is used to estimate the multidimensional integrals defining the considered indices.

\subsection{The SMAA-Choquet method}

As shown in Eq. (1), the application of the Choquet integral preference model asks, on one hand, that the evaluations of the alternatives on the considered criteria are given on the same scale and, on the other hand, that a capacity $\mu$ is given.

Regarding the first point, in order to build a scale common to all the evaluations of the alternatives, in Angilella et al. (2015) an heuristic, that we shall briefly recall in the following, has been proposed.

For each criterion $g_{i} \in G, \alpha$ different values $x_{1}, \ldots, x_{\alpha}$ are uniformly sampled in the interval [0,1], where $\alpha$ is the number of different evaluations got on criterion $g_{j}$ by the alternatives in $A$, that is $\mid\left\{x \in \mathbb{R}: g_{i}\left(a_{k}\right)=\right.$ $\left.x, a_{k} \in A\right\} \mid=\alpha$. Then, after ordering the $\alpha$ real numbers in an increasing way, that is $x_{i(1)}<x_{i(2)}<\ldots<$ $x_{i(\alpha)}$, one has to assign the value $x_{i(h)}, h=1, \ldots, \alpha$, to the alternative(s) having the $h$-th evaluation in an increasing way with respect to the preferences of the DM on criterion $g_{i}$. For example, let us consider five alternatives evaluated on criterion $g_{i}$ as shown in the first line of Table 5 .

Table 5: An example of the scale construction

\begin{tabular}{|c|ccccc|}
\hline Criterion/Alternative & $a_{1}$ & $a_{2}$ & $a_{3}$ & $a_{4}$ & $a_{5}$ \\
\hline$g_{i}$ & 3 & 5 & 3 & 4 & 6 \\
\hline Constructed value & 0.1270 & 0.9058 & 0.1270 & 0.8147 & 0.9134 \\
\hline
\end{tabular}

After sampling four different real numbers, $x_{1}=0.8147, x_{2}=0.9058, x_{3}=0.1270$ and $x_{4}=0.9134$, and after ordering them in an increasing way, $x_{i(1)}=0.1270<x_{i(2)}=0.8147<x_{i(3)}=0.9058<x_{i(4)}=0.9134$, these values are assigned to the alternatives $a_{1}, \ldots, a_{5}$ as shown in the second line of Table 5. Applying the same procedure for each of the $n$ criteria, a scale common to the evaluations of the alternatives on the considered criteria is built. 
Regarding the second point, in order to get a capacity $\mu$ necessary for the application of the Choquet integral preference model, the DM has to provide a direct or an indirect preference information. In the direct case, the DM has to provide a value for each subset of criteria $T \subseteq G(\mu(T))$ representing the importance of the set of criteria $T$. Since the DM, in general, is not able to provide this information, the indirect preference information is more used in practice (Jacquet-Lagrèze and Siskos, 2001). In the indirect case, the DM has to provide some preferences in terms of comparisons between alternatives (for example, $a_{h}$ is preferred to $a_{k}$ or $a_{h}$ and $a_{k}$ are indifferent) or comparison between importance of criteria and interaction between criteria (for example, $g_{i}$ is more important than $g_{j}$ or criteria $g_{i}$ and $g_{j}$ are positively interacting) from which a capacity compatible with these preferences can be inferred. After that these preferences are translated to constraints, the space of the 2-additive Möbius representations of capacities $\mu$ compatible with the preferences provided by the DM is defined by the following set of constraints,

$$
\begin{aligned}
& \begin{array}{l}
\varphi\left(\left\{g_{i}\right\}\right) \geq \varphi\left(\left\{g_{j}\right\}\right)+\varepsilon, \quad \text { if criterion } i \text { is more important than criterion } j, \text { with } i, j \\
\varphi\left(\left\{g_{i}, g_{j}\right\}\right) \geq \varepsilon, \quad \text { if criteria } i \text { and } j \text { are positively interacting, with } i, j \in G,
\end{array} \\
& \varphi\left(\left\{g_{i}, g_{j}\right\}\right) \leq-\varepsilon, \quad \text { if criteria } i \text { and } j \text { are negatively interacting, with } i, j \in G \text {, } \\
& \left.\begin{array}{ll}
C_{\mu}\left(a_{h}\right) \geq C_{\mu}\left(a_{k}\right)+\varepsilon, & \text { if } a_{h} \text { is preferred to } a_{k} \\
C_{\mu}\left(a_{h}\right)=C_{\mu}\left(a_{k}\right), & \text { if } a_{h} \text { is indifferent to } a_{k}
\end{array}\right\} E^{A} \\
& m(\{\emptyset\})=0, \quad \sum_{g_{i} \in G} m\left(\left\{g_{i}\right\}\right)+\sum_{\left\{g_{i}, g_{j}\right\} \subseteq G} m\left(\left\{g_{i}, g_{j}\right\}\right)=1, \\
& m\left(\left\{g_{i}\right\}\right) \geq 0, \forall g_{i} \in G, \\
& \left.m\left(\left\{g_{i}\right\}\right)+\sum_{g_{j} \in T} m\left(\left\{g_{i}, g_{j}\right\}\right) \geq 0, \forall g_{i} \in G \text { and } \forall T \subseteq G \backslash\left\{g_{i}\right\}\right\} \\
& \} E^{C} E^{D M}
\end{aligned}
$$

where $\varepsilon$ is an auxiliary variable used to convert the strict inequalities in weak inequalities. For example, the constraint $\varphi\left(\left\{g_{i}\right\}\right)>\varphi\left(\left\{g_{j}\right\}\right)$, translating the preference of criterion $g_{i}$ over criterion $g_{j}$, is converted into the weak constraint $\varphi\left(\left\{g_{i}\right\}\right) \geq \varphi\left(\left\{g_{j}\right\}\right)+\varepsilon$. If $E^{D M}$ is feasible and $\varepsilon^{*}>0$, where $\varepsilon^{*}=\max \varepsilon$ subject to $E^{D M}$, then there exists at least one capacity compatible with the preferences provided by the DM.

Considering the two aspects simultaneously, many common scales can be built by using the proposed heuristic and many capacities compatible with the preferences provided by the DM can be inferred. For this reason, the SMAA-Choquet method proposes to take into account at the same time a plurality of common scales and a plurality of compatible capacities by means of an iterative procedure. At each iteration, a common scale is built by using the procedure described above and, therefore, the existence of a capacity compatible with the preferences provided by the DM has to be checked by solving the previous LP problem. We have to distinguish the case in which the DM is able to provide preference information in terms of comparisons between alternatives $\left(E^{A} \neq \emptyset\right)$ from the case in which $(\mathrm{s})$ he is not able to do it $\left(E^{A}=\emptyset\right)$, since the sampling procedure depends on it:

- In the first case, since constraints translating the preferences of the DM on the considered alternatives 
are dependent on the evaluations of the same alternatives on the considered criteria, that are replaced by the values obtained in the common scale, the set of constraints $E^{D M}$ will vary depending on the built common scale. Consequently, it is possible that for a certain built common scale none capacity compatible with the preferences provided by the DM there exists. If there exists a compatible capacity, then it is stored. The procedure of building the common scale ends when a satisfying number of scales, for example 10,000, and the corresponding 10,000 capacities compatible with the preferences provided by the DM have been stored.

- In the second case, the set of constraints $E^{D M}$ will be independent on the built common scale. Consequently, the procedure used to build the common scale and the procedure used to sample a capacity compatible with the preferences provided by the DM are independent. Therefore, 10,000 common scales are built by using the suggested heuristic, while 10,000 compatible capacities can be sampled by using the Hit-And-Run method (Smith, 1984; Tervonen et al., 2013) since the set of compatible capacities defined by the constraints in $E^{D M}$ is convex.

In both cases, 10,000 pairs (scale, capacity) have been stored and for each of them the Choquet integral of the alternatives at hand and their ranking can be obtained. After computing the corresponding 10,000 rankings of the alternatives, the rank acceptability indices and pairwise winning indices described in section 3.2 can be computed. More details on the SMAA-Choquet method can be found in Angilella et al. (2015).

\section{SMAA-Choquet applied to the ORC simple scoring}

In this section we apply the SMAA-Choquet method to the "53th Syracuse - Malta" regatta ${ }^{8}$ which length is 82.4 miles. At this end, we shall consider 13 sailboats participating to the regatta that will be the considered alternatives, while the 5 scoring options will be the evaluation criteria. In the following, we shall denote the five scoring options by $g_{T o D}, g_{T o T}, g_{P L}, g_{T N}$ and $g_{G P H}$ and, consequently, the set of criteria will be denoted by $G=\{T o D, T o T, P L, T N, G P H\}$. The corrected time computed by the five considered scoring options will be the evaluations of the alternatives (the different sailboats) with respect to the considered criteria and they are provided in Table 6 .

To apply the SMAA-Choquet method to this particular problem, the preference information was collected by a DM being an expert on the Syracuse-Malta regatta. We asked him to provide us some preference information of the nature shown in Section 3.3. On one hand he was confident enough in providing preference information on the considered criteria, while he was not sure in the comparisons between the alternatives at hand since he did not know very well their design characteristics. Consequently, $E^{A}=\emptyset$. In the following, we shall give in detail the statements provided by the expert:

\footnotetext{
${ }^{8}$ Official website of the Siracusa-Malta regatta: http://www.rmyc.org/races/archive/?id=115\&page=4
} 
Table 6: Table of the evaluations of the different sailboats expressed in seconds for each simple scoring option. For the sake of simplicity, with respect to Triple Number scoring option, we averaged the three evaluations with respect to Low, Medium and High wind speed

\begin{tabular}{|l|l|c|c|c|c|c|}
\hline & Sailboat / Scoring Option & $g_{T o D}$ & $g_{T o T}$ & $g_{P L}$ & $g_{T N}$ & $g_{G P H}$ \\
\hline$a_{1}$ & OTTOVOLANTE & 23,530 & 73,144 & 54,225 & 88,191 & 22,549 \\
\hline$a_{2}$ & JUNO OILTANKING & 25,193 & 76,546 & 57,768 & 92,471 & 24,773 \\
\hline$a_{3}$ & WOLVERINE & 26,744 & 74,856 & 55,853 & 89,827 & 25,533 \\
\hline$a_{4}$ & NAUTILUS QQ7 & 26,518 & 77,374 & 57,958 & 93,400 & 25,760 \\
\hline$a_{5}$ & UNICA & 30,135 & 81,139 & 61,187 & 97,610 & 29,047 \\
\hline$a_{6}$ & ARTIE RTFX & 30,194 & 81,358 & 62,087 & 98,029 & 29,683 \\
\hline$a_{7}$ & RICOMINCIO DA TRE & 30,954 & 78,812 & 59,338 & 94,642 & 29,859 \\
\hline$a_{8}$ & DREAMER TECH & 31,212 & 87,667 & 64,689 & 105,588 & 30,339 \\
\hline$a_{9}$ & XP-ACT & 32,171 & 85,014 & 65,104 & 102,633 & 31,504 \\
\hline$a_{10}$ & SQUALO BIANCO & 33,599 & 82,026 & 61,998 & 98,540 & 32,495 \\
\hline$a_{11}$ & EMILE GALLE' & 33,672 & 83,070 & 63,999 & 100,020 & 32,815 \\
\hline$a_{12}$ & PROFILO & 34,403 & 83,520 & 64,044 & 100,688 & 33,629 \\
\hline$a_{13}$ & MAYDA & 34,341 & 84,195 & 63,945 & 101,248 & 33,872 \\
\hline
\end{tabular}

- Information in terms of importance of criteria:

1. the Performance Line is considered the most important scoring option since it gives the best approximation $^{9}$ of the time necessary to each sailboat to complete the race. This preference is translated to the constraints $\varphi\left(\left\{g_{P L}\right\}\right)>\varphi\left(\left\{g_{i}\right\}\right)$ for all $i \in G \backslash\{P L\}$;

2. the Time on Distance and the Time on Time scoring options are the most adopted in long and short races, respectively. In a comparison between the two criteria, the DM thinks that they have the same importance. This piece of information is translated to the constraint $\varphi\left(\left\{g_{T o D}\right\}\right)=$ $\varphi\left(\left\{g_{T o T}\right\}\right) ;$

3. as previously observed, the corrected time computed by the Triple Number scoring option depends only on the speed of the wind that in a long race, such the Syracuse - Malta is, can not be assumed as constant, while it does not consider the length of the course of the race. Since, instead, the corrected time computed by the Time on Distance scoring option depends on the covered distance, the DM considers the Time on Distance scoring option more important than the Triple Number one. The related constraint is $\varphi\left(\left\{g_{T o D}\right\}\right)>\varphi\left(\left\{g_{T N}\right\}\right)$;

\footnotetext{
${ }^{9}$ The Performance Line is a linearization of the Performance Curve which is a function that, given the wind speed, gives back the speed of the sailboat.
} 
4. a DM can argue that the General Purpouse Handicap scoring option gives less information on the race with respect to the others and, therefore, we can add the following constraints: $\varphi\left(\left\{g_{G P H}\right\}\right)<\varphi\left(\left\{g_{i}\right\}\right)$ for all $i \in G \backslash\{G P H\}$.

- Information in terms of interaction between criteria:

1. since, as already stated above, the Time on Distance scoring option and the Time on Time scoring option take into account different aspects of the considered race, a sailboat presenting good values of the corrected time for both scoring options will be particularly appreciated. Therefore the two criteria $g_{T o D}$ and $g_{T o T}$ present a positive interaction. The constraint translating this piece of preference information is $\varphi\left(\left\{g_{T o D}, g_{T o T}\right\}\right)>0$;

2. the formulas used in the Time on Distance and in the General Purpouse Handicap scoring options differ only in the coefficients used to compute the corrected time. For this reason, the two criteria can be considered as negatively interacting because, in general, a good performance on one of the two criteria can be expected when there is a good performance on the other criterion. The constraint translating this piece of preference information is $\varphi\left(\left\{g_{T o D}, g_{G P H}\right\}\right)<0$;

3. for the same reasons expressed in the previous item, the Time on Time scoring option and the Triple Number one present a negative interaction. The constraint $\varphi\left(\left\{g_{T o T}, g_{T N}\right\}\right)<0$ is used to translate this piece of preference information;

4. the Performance Line scoring option takes into account aspects already considered both in the Time on Distance and Time on Time scoring options. Consequently, the DM thinks that the two pairs of criteria $\left\{g_{P L}, g_{T o D}\right\}$ and $\left\{g_{P L}, g_{T o T}\right\}$ are negatively interacting. The constraints $\varphi\left(\left\{g_{T o D}, g_{P L}\right\}\right)<0$ and $\varphi\left(\left\{g_{T o T}, g_{P L}\right\}\right)<0$ are therefore used to translate this piece of preference information.

Summing up, the set of constraints translating the preferences of the DM is the following:

$$
\begin{aligned}
& \varphi\left(\left\{g_{P L}\right\}\right) \geq \varphi\left(\left\{g_{T o D}\right\}\right)+\varepsilon \\
& \varphi\left(\left\{g_{T o D}\right\}\right)=\varphi\left(\left\{g_{T o T}\right\}\right) \\
& \varphi\left(\left\{g_{T o T}\right\}\right) \geq \varphi\left(\left\{g_{T N}\right\}\right)+\varepsilon \\
& \varphi\left(\left\{g_{T N}\right\}\right) \geq \varphi\left(\left\{g_{G P H}\right\}\right)+\varepsilon \\
& \varphi\left(g_{T o T}, g_{P L}\right) \leq-\varepsilon \\
& \varphi\left(\left\{g_{T o D}, g_{T o T}\right\}\right) \geq \varepsilon \\
& \varphi\left(\left\{g_{T o D}, g_{G P H}\right\}\right) \leq-\varepsilon \\
& \varphi\left(\left\{g_{T o T}, g_{T N}\right\}\right) \leq-\varepsilon \\
& \varphi\left(\left\{g_{T o D}, g_{P L}\right\}\right) \leq-\varepsilon
\end{aligned}
$$


Based on the preference information provided by the DM, and observing that she did not provide any preference in terms of comparisons between alternatives, we apply the SMAA-Choquet method by sampling 10,000 pairs (common scale, capacity) computing for each of them the Choquet integral of the 13 alternatives. From the computational point of view, the method has been implemented in Matlab environment and executed on a computer with an Intel Core 2 Quad 2.0 GHz and 4 Gb of RAM. Consequently, the rank acceptability indices and the pairwise winning indices described in Section 3.2 have been computed and reported in Tables 7 and 8, respectively.

Table 7: Rank Acceptability Indices of the considered sailboats expressed in percentage

\begin{tabular}{|c|c|c|c|c|c|c|c|c|c|c|c|c|c|c|}
\hline & Sailboat & $b_{k}^{1}$ & $b_{k}^{2}$ & $b_{k}^{3}$ & $b_{k}^{4}$ & $b_{k}^{5}$ & $b_{k}^{6}$ & $b_{k}^{7}$ & $b_{k}^{8}$ & $b_{k}^{9}$ & $b_{k}^{10}$ & $b_{k}^{11}$ & $b_{k}^{12}$ & $b_{k}^{13}$ \\
\hline$a_{1}$ & OTTOVOLANTE & 100 & 0 & 0 & 0 & 0 & 0 & 0 & 0 & 0 & 0 & 0 & 0 & 0 \\
\hline$a_{2}$ & JUNO OILTANKING & 0 & 39.64 & 60.36 & 0 & 0 & 0 & 0 & 0 & 0 & 0 & 0 & 0 & 0 \\
\hline$a_{3}$ & WOLVERINE & 0 & 60.36 & 38.94 & 0.70 & 0 & 0 & 0 & 0 & 0 & 0 & 0 & 0 & 0 \\
\hline$a_{4}$ & NAUTILUS QQ7 & 0 & 0 & 0.70 & 99.30 & 0 & 0 & 0 & 0 & 0 & 0 & 0 & 0 & 0 \\
\hline$a_{5}$ & UNICA & 0 & 0 & 0 & 0 & 48.66 & 51.34 & 0 & 0 & 0 & 0 & 0 & 0 & 0 \\
\hline$a_{6}$ & ARTIE RTFX & 0 & 0 & 0 & 0 & 0 & 1.17 & 97.53 & 1.30 & 0 & 0 & 0 & 0 & 0 \\
\hline$a_{7}$ & RICOMINCIO DA TRE & 0 & 0 & 0 & 0 & 51.34 & 47.49 & 1.17 & 0 & 0 & 0 & 0 & 0 & 0 \\
\hline$a_{8}$ & DREAMER TECH & 0 & 0 & 0 & 0 & 0 & 0 & 0 & 0 & 2.95 & 17.98 & 25.95 & 32.04 & 21.08 \\
\hline$a_{9}$ & $\mathrm{XP}-\mathrm{ACT}$ & 0 & 0 & 0 & 0 & 0 & 0 & 0 & 0 & 1.29 & 11.06 & 20.58 & 28.73 & 38.34 \\
\hline$a_{10}$ & SQUALO BIANCO & 0 & 0 & 0 & 0 & 0 & 0 & 1.30 & 98.7 & 0 & 0 & 0 & 0 & 0 \\
\hline$a_{11}$ & EMILE GALLÉ & 0 & 0 & 0 & 0 & 0 & 0 & 0 & 0 & 89.39 & 8.99 & 1.43 & 0.19 & 0 \\
\hline$a_{12}$ & PROFILO & 0 & 0 & 0 & 0 & 0 & 0 & 0 & 0 & 0 & 12.51 & 31.91 & 22.68 & 32.90 \\
\hline$a_{13}$ & MAYDA & 0 & 0 & 0 & 0 & 0 & 0 & 0 & 0 & 6.37 & 49.46 & 20.13 & 16.36 & 7.68 \\
\hline
\end{tabular}

Table 8: Pairwise Winning Indices for each pair of alternatives expressed in percentage

\begin{tabular}{|c|c|c|c|c|c|c|c|c|c|c|c|c|c|c|}
\hline & Sailboat / Sailboat & $a_{1}$ & $a_{2}$ & $a_{3}$ & $a_{4}$ & $a_{5}$ & $a_{6}$ & $a_{7}$ & $a_{8}$ & $a_{9}$ & $a_{10}$ & $a_{11}$ & $a_{12}$ & $a_{13}$ \\
\hline$a_{1}$ & OTTOVOLANTE & 0 & 100 & 100 & 100 & 100 & 100 & 100 & 100 & 100 & 100 & 100 & 100 & 100 \\
\hline$a_{2}$ & JUNO OILTANKING & 0 & 0 & 39.64 & 100 & 100 & 100 & 100 & 100 & 100 & 100 & 100 & 100 & 100 \\
\hline$a_{3}$ & WOLVERINE & 0 & 60.36 & 0 & 99.3 & 100 & 100 & 100 & 100 & 100 & 100 & 100 & 100 & 100 \\
\hline$a_{4}$ & NAUTILUS QQ7 & 0 & 0 & 0.70 & 0 & 100 & 100 & 100 & 100 & 100 & 100 & 100 & 100 & 100 \\
\hline$a_{5}$ & UNICA & 0 & 0 & 0 & 0 & 0 & 100 & 48.66 & 100 & 100 & 100 & 100 & 100 & 100 \\
\hline$a_{6}$ & ARTIE RTFX & 0 & 0 & 0 & 0 & 0 & 0 & 1.17 & 100 & 100 & 98.70 & 100 & 100 & 100 \\
\hline$a_{7}$ & RICOMINCIO DA TRE & 0 & 0 & 0 & 0 & 51.34 & 98.83 & 0 & 100 & 100 & 100 & 100 & 100 & 100 \\
\hline$a_{8}$ & DREAMER TECH & 0 & 0 & 0 & 0 & 0 & 0 & 0 & 0 & 62.92 & 0 & 3.58 & 52.94 & 30.24 \\
\hline$a_{9}$ & $\mathrm{XP}-\mathrm{ACT}$ & 0 & 0 & 0 & 0 & 0 & 0 & 0 & 37.08 & 0 & 0 & 2.18 & 44.78 & 24.19 \\
\hline$a_{10}$ & SQUALO BIANCO & 0 & 0 & 0 & 0 & 0 & 1.30 & 0 & 100 & 100 & 0 & 100 & 100 & 100 \\
\hline$a_{11}$ & EMILE GALLÉ & 0 & 0 & 0 & 0 & 0 & 0 & 0 & 96.42 & 97.82 & 0 & 0 & 100 & 93.34 \\
\hline$a_{12}$ & PROFILO & 0 & 0 & 0 & 0 & 0 & 0 & 0 & 47.06 & 55.22 & 0 & 0 & 0 & 21.75 \\
\hline$a_{13}$ & MAYDA & 0 & 0 & 0 & 0 & 0 & 0 & 0 & 69.76 & 75.81 & 0 & 6.66 & 78.25 & 0 \\
\hline
\end{tabular}

Looking at Table 7 one can see that, apart from OTTOVOLANTE that is the best among the considered sailboats in each case, each sailboat can fill different positions depending on the built common scale and on the sampled capacity compatible with the preferences of the DM. For example, JUNO OILTANKING can fill the second and the third positions with a frequency of the $39.64 \%$ and $60.36 \%$, respectively. WOLVERINE 
is the sailboat filling the second position more frequently since $b_{3}^{2}=60.36 \%$, NAUTILUS QQ7 is almost always in the fourth position $\left(b_{4}^{4}=99.3 \%\right)$ while one among UNICA and RICOMINCIO DA TRE is the fifth among the considered sailboats since $b_{5}^{5}=48.66 \%$ and $b_{7}^{5}=51.34 \%$. Looking at the last positions in the ranking, one can observe that the worst is one between DREAMER TECH, XP-ACT, PROFILO and MAYDA. Indeed, only these four sailboats can fill the last position in the ranking and, moreover, their best possible rank position is the $9^{t h}$.

Analogously, looking at Table 8 one can observe that MAYDA is the best among the ones that can fill the last position since it is preferred to the other three with a frequency at least equal to the $69.76 \%$; PROFILO is preferred with a frequency greater than the $50 \%$ only to XP-ACT $\left(p\left(a_{12,9}\right)=55.22 \%\right)$ while DREAMER TECH is preferred to XP-ACT and PROFILO with a frequency of the $62.92 \%$ and $52.94 \%$. The worst among the four considered sailboats appears to be XP-ACT since the other three sailboats are preferred to it with a frequency at least equal to the $55.22 \%$.

Since the DM is interested in getting a unique ranking of the alternatives at hand, we need to consider only one among the built common scales and only one among the capacities compatible with the preferences provided by the DM. As explained in section 3.3, to each built common scale $S_{k}$ corresponds a value $\varepsilon_{k}^{*}$ obtained by solving the corresponding LP problem. Therefore, among the built common scales, we consider the most discriminant one, that is the common scale $S_{D M}$ such that $\varepsilon_{D M}^{*}=\max _{k} \varepsilon_{k}^{*}$. The most discriminant common scale is shown in Table 9.

Table 9: Most discriminant built common scale

\begin{tabular}{|l|r|r|r|r|r|}
\hline Sailboat / Criterion & ToD & ToT & PL & TN & GPH \\
\hline OTTOVOLANTE & 0.9016 & 0.8564 & 0.9218 & 0.9102 & 0.9028 \\
\hline JUNO OILTANKING & 0.8089 & 0.6022 & 0.5966 & 0.7698 & 0.5542 \\
\hline WOLVERINE & 0.6412 & 0.7925 & 0.6205 & 0.7961 & 0.5457 \\
\hline NAUTILUS QQ7 & 0.7864 & 0.5979 & 0.5555 & 0.7647 & 0.5422 \\
\hline UNICA & 0.6393 & 0.2760 & 0.2098 & 0.6315 & 0.4841 \\
\hline ARTIE RTFX & 0.5703 & 0.1967 & 0.1719 & 0.4595 & 0.4436 \\
\hline RICOMINCIO DA TRE & 0.5583 & 0.3777 & 0.5085 & 0.7245 & 0.3698 \\
\hline DREAMER TECH & 0.4457 & 0.0479 & 0.0540 & 0.0367 & 0.3545 \\
\hline XP-ACT & 0.4260 & 0.0557 & 0.0055 & 0.0523 & 0.2145 \\
\hline SQUALO BIANCO & 0.3793 & 0.1898 & 0.2017 & 0.2828 & 0.1668 \\
\hline EMILE GALLE' & 0.3322 & 0.1217 & 0.1646 & 0.2346 & 0.1560 \\
\hline PROFILO & 0.0285 & 0.0658 & 0.1158 & 0.1788 & 0.1367 \\
\hline MAYDA & 0.1405 & 0.0641 & 0.1672 & 0.1736 & 0.0001 \\
\hline
\end{tabular}

At the same time, among the capacities compatible with the preferences provided by the DM, we shall consider their barycenter that is their average component by component (see Table 10). 
Table 10: The Möbius representation of the barycenter of the capacities compatible with the preferences provided by the DM

\begin{tabular}{|c|c|c|c|c|c|c|c|c|c|c|}
\hline$m\left(g_{T o D}\right)$ & $m\left(g_{T o T}\right)$ & $m\left(g_{P L}\right)$ & $m\left(g_{T N}\right)$ & $m\left(g_{G P H}\right)$ & $m\left(g_{T o D}, g_{T o T}\right)$ & $m\left(g_{T o D}, g_{P L}\right)$ & $m\left(g_{T o D}, g_{G P H}\right)$ & $m\left(g_{T o T}, g_{P L}\right)$ & $m\left(g_{T o T}, g_{T N}\right)$ & $m\left(g_{P L}, g_{T N}\right)$ \\
\hline 0.2626 & 0.2803 & 0.3836 & 0.2034 & 0.1290 & 0.078 & -0.0963 & 0.0093 & -0.0645 & -0.0952 & 0.0017 \\
\hline
\end{tabular}

By using the common scale and the Möbius representation of the barycenter of the compatible capacities shown in Tables 9 and 10, we can compute the Choquet integral of the 13 sailboats obtaining, therefore, their ranking shown in Table 11.

Table 11: Ranking of the sailboats considerig the most discriminat scale and the Möbius representation of the barycenter of the compatible capacities

\begin{tabular}{|l|c|}
\hline Position & Sailboat \\
\hline $1^{\text {st }}$ & OTTOVOLANTE \\
$2^{\text {nd }}$ & WOLVERINE \\
$3^{\text {rd }}$ & JUNO OILTANKING \\
$4^{\text {th }}$ & NAUTILUS QQ7 \\
$5^{t h}$ & RICOMINCIO DA TRE \\
$6^{t h}$ & UNICA \\
$7^{t h}$ & ARTIE RTFX \\
$8^{t h}$ & SQUALO BIANCO \\
$9^{t h}$ & EMILE GALLÉ \\
$10^{t h}$ & DREAMER TECH \\
$11^{t h}$ & XP-ACT \\
$12^{t h}$ & MAYDA \\
$13^{t h}$ & PROFILO \\
\hline
\end{tabular}

One can observe that the recommendations obtained by the rank acceptability indices shown in Table 7 are almost confirmed. Indeed, the first nine positions are filled by the sailboats presenting the highest rank acceptability index for that position (for example WOLVERINE is second with a frequency of the $60.36 \%$ while EMILE GALLÉ fills the ninth position with a frequency equal to the 89.39\%). The last four positions (from the $10^{\text {th }}$ to the $13^{\text {th }}$ ) are filled by the DREAMER TECH, XP-ACT, MAYDA and PROFILO being the four sailboats presenting the highest rank acceptability indices for these positions. 


\section{Conclusions}

In general, different scoring options are used to compute the corrected time of the sailboats involved in the regattas. Each scoring option takes into account different physical characteristics of the sailboats as well as different weather aspects occurring during the race. For this reason, the values of the corrected time computed by the different scoring options will be different and, moreover, they will be expressed on different scales.

In this paper, we proposed the application of the SMAA-Choquet method (Angilella et al., 2015) to compare the performances of different sailboats in sailing regattas. In particular, we showed the results of the application of the SMAA-Choquet method to the "53th Syracuse - Malta" regatta, considering 13 sailboats participating to the race as the alternatives at hand and five different scoring options used to compute the corrected time as the evaluation criteria on which the alternatives are evaluated. The application of the SMAA-Choquet method permits, on one hand, to compute a scale common to all the evaluations so that the values of the corrected time computed by the different scoring options become comparable and, on the other hand, it permits to take into account the possible positive and negative interactions among the five considered scoring options highlighted by the Decision Maker (DM). Moreover, the Stochastic Multiobjective Acceptability Analysis (SMAA) (Lahdelma et al., 1998; Tervonen and Figueira, 2008) permits to take into account simultaneously all the built common scales and all the capacities compatible with the preference information provided by the DM.

In future works we plan to apply the SMAA-Choquet method to other regatta races and, moreover, we would like to apply this method as well as other aggregation methods such as PROMETHEE (Brans and Vincke, 1985) and ELECTRE (Figueira et al., 2013; Roy, 1996), to deal with other sport problems.

\section{Acknowledgments}

The first, the third and the fourth authors wish also to acknowledge funding by the "FIR of the University of Catania BCAEA3 New developments in Multiple Criteria Decision Aiding (MCDA) and their application to territorial competitiveness"

\section{References}

S. Angilella, S. Greco, F. Lamantia, and B. Matarazzo. Assessing non-additive utility for multicriteria decision aid. European Journal of Operational Research, 158(3):734-744, 2004.

S. Angilella, S. Greco, and B. Matarazzo. Non-additive robust ordinal regression: A multiple criteria decision model based on the Choquet integral. European Journal of Operational Research, 201(1):277-288, 2010. 
S. Angilella, S. Corrente, and S. Greco. Stochastic multiobjective acceptability analysis for the Choquet integral preference model and the scale construction problem. European Journal of Operational Research, 240(1):172-182, 2015.

C. Bana e Costa and J.-C. Vansnick. MACBETH-An interactive path towards the construction of cardinal value functions. International Transactions in Operational Research, 1(4):489-500, 1994.

F.H. Barron and B.E. Barrett. Decision quality using ranked attribute weights. Management Science, 42 (11):1515-1523, 1996.

J.P. Brans and Ph. Vincke. A preference ranking organisation method: The PROMETHEE method for MCDM. Management Science, 31(6):647-656, 1985.

A. Chateauneuf and J.Y. Jaffray. Some characterizations of lower probabilities and other monotone capacities through the use of Möbius inversion. Mathematical Social Sciences, 17:263-283, 1989.

G. Choquet. Theory of capacities. Annales de l'Institut Fourier, 5:131-295, 1953.

S. Corrente, S. Greco, and A. Ishizaka. Analytical Hierarchy Process and Choquet integral within nonadditive robust ordinal regression. Omega, 2016. DOI:10.1016/j.omega.2015.07.003.

S. Dadelo, Z. Turskis, E.K. Zavadskas, and R. Dadeliene. Multi-criteria assessment and ranking system of sport team formation based on objective-measured values of criteria set. Expert Systems with Applications, 41(14):6106-6113, 2014.

P.K. Dey, D.N. Ghosh, and A.C. Mondal. A MCDM approach for evaluating bowlers performance in IPL. Journal of Emerging Trends in Computing and Information Sciences, 2(11):563-73, 2011.

W. Edwards. How to use multiattribute utility measurement for social decisionmaking. IEEE Transactions on Systems, Man, and Cybernetics, 7(5):326-340, 1977.

J.R. Figueira, S. Greco, B. Roy, and R. Słowiński. An overview of ELECTRE methods and their recent extensions. Journal of Multi-Criteria Decision Analysis, 20:61-85, 2013.

J.R. Figueira, S. Greco, and M. Ehrgott. Multiple Criteria Decision Analysis: State of the Art Surveys. Springer, Berlin, 2016.

Y. Gerchak. Operations Research in sports. In S.M. Pollock, M.H. Rothkopf, and A. Barnett, editors, Handbooks in Operations Research and Management Science, vol. 6, pages 507-527, 1994.

I. Gilboa and D. Schmeidler. Additive representations of non-additive measures and the Choquet integral. Annals of Operations Research, 52:43-65, 1994. 
M. Grabisch. The application of fuzzy integrals in multicriteria decision making. European Journal of Operational Research, 89:445-456, 1996.

M. Grabisch. k-order additive discrete fuzzy measures and their representation. Fuzzy Sets and Systems, 92:167-189, 1997.

S. Greco, B. Matarazzo, and R. Slowinski. Rough sets theory for multicriteria decision analysis. European Journal of Operational Research, 129(1):1-47, 2001.

C.L. Hwang and K. Yoon. Multiple Attribute Decision Making. Springer Verlag, New York, 1981.

A. Ishizaka and P. Nemery. Multi-Criteria Decision Analysis: methods and software. John Wiley \& Sons, 2013.

J. Jablonsky. Multicriteria Analysis of Classification in Athletic Decathlon. Multiple Criteria Decision Making, 7:112-120, 2012.

E. Jacquet-Lagrèze and Y. Siskos. Preference disaggregation: 20 years of MCDA experience. European Journal of Operational Research, 130(2):233-245, 2001.

R.L. Keeney and H. Raiffa. Decisions with multiple objectives: Preferences and value tradeoffs. J. Wiley, New York, 1993.

G. Kendall, S. Knust, C.C. Ribeiro, and S. Urrutia. Scheduling in sports: An annotated bibliography. Computers \& Operations Research, 37(1):1-19, 2010.

R. Lahdelma and P. Salminen. SMAA-2: Stochastic Multicriteria Acceptability Analysis for group decision making. Operations Research, 49(3):444-454, 2001.

R. Lahdelma, J. Hokkanen, and P. Salminen. SMAA - Stochastic Multiobjective Acceptability Analysis. European Journal of Operational Research, 106(1):137-143, 1998.

P. Leskinen, J. Viitanen, A. Kangas, and J. Kangas. Alternatives to incorporate uncertainty and risk attitude in multicriteria evaluation of forest plans. Forest Science, 52(3):304-312, 2006.

J.L. Marichal and M. Roubens. Determination of weights of interacting criteria from a reference set. European Journal of Operational Research, 124(3):641-650, 2000.

F. Modave and M. Grabisch. Preference representation by the Choquet integral: The commensurability hypothesis. Proceedings of the Seventh International Conference IPMU Paris, July 610, 1998, pages 164-171, 1998.

C.M. Mottley. The application of Operations-Research methods to athletic games. Journal of the Operations Research Society of America, 2(3):335-338, 1954. 
S. Murofushi and T. Soneda. Techniques for reading fuzzy measures (III): interaction index. 9th Fuzzy Systems Symposium, Sapporo, Japan, pages 693-696, 1993.

D.L. Olson. Comparison of three multicriteria methods to predict known outcomes. European Journal of Operational Research, 130(3):576-587, 2001.

G.C. Rota. On the foundations of combinatorial theory. I. Theory of Möbius functions. Wahrscheinlichkeitstheorie und Verwandte Gebiete, 2:340-368, 1964.

B. Roy. Multicriteria Methodology for Decision Aiding. Kluwer, Dordrecht, 1996.

T. Saaty. How to make a decision: the Analytic Hierarchy Process. European Journal of Operational Research, 48(1):9-26, 1990.

L.S. Shapley. A value for n-person games. In Tucker A. W. Kuhn, H. W., editor, Contributions to the Theory of Games II, page 307. Princeton University Press, Princeton, 1953.

R.L. Smith. Efficient Monte Carlo procedures for generating points uniformly distributed over bounded regions. Operations Research, 32:1296-1308, 1984.

J.C.C.B. Soares de Mello, J. Benício, L. Bragança, and V. Guimarães. The MACBETH method for ranking olympic sports: a complementary analysis for the DEA efficiency. Proceedings of the fourth International Conference on Mathematics in Sport, Leuven, June 5-7, 2013, pages 325-333, 2013.

M. Sugeno. Theory of fuzzy integrals and its applications. Ph.D. Thesis, Tokyo Institute of Technology, 1974.

T. Tervonen and J.R. Figueira. A survey on stochastic multicriteria acceptability analysis methods. Journal of Multi-Criteria Decision Analysis, 15(1-2):1-14, 2008.

T. Tervonen, G. Van Valkenhoef, N. Bastürk, and D. Postmus. Hit-and-run enables efficient weight generation for simulation-based multiple criteria decision analysis. European Journal of Operational Research, 224:552-559, 2013.

M.B. Wright. 50 years of OR in sport. Journal of the Operational Research Society, 60(1):S161-S168, 2009. 